\title{
The Effect of Post-Learning Wakeful Rest on the Retention of Second Language Learning Material over the Long Term
}

\author{
Markus Martini $^{1} \cdot$ Benjamin Riedlsperger $^{1} \cdot$ Thomas Maran $^{1} \cdot$ Pierre Sachse $^{1}$ \\ Published online: 16 December 2017 \\ (C) The Author(s) 2017. This article is an open access publication
}

\begin{abstract}
Evidence exists that a brief period of wakeful rest after learning supports memory retention, while interference after learning weakens memory retention. We tested this so-called resting effect for second language learning material. In two counterbalanced within-subject designs, participants were involved in two identical successive learning phases. In each phase, they learned one of two second language stories, immediately followed by a recall phase in the first language. The critical manipulation took place after the immediate recall. Here, participants either rested or worked on a distractor task for $8 \mathrm{~min}$. In Experiment 1, participants worked on a spot-the-difference task (visual distractor condition), and in Experiment 2, they read a New York Times article (verbal distractor condition). After 7 days, all participants were again invited to a second experimental session, where a surprise free recall test took place. Our results showed that second language story retention over a period of 7 days was not supported by a brief period of wakeful rest following learning and recall, independent of the distractor condition. Our findings contribute to an important characterization of the wakeful resting effect in younger adults, which is discussed in the light of existing studies.
\end{abstract}

Keywords Wakeful resting $\cdot$ Consolidation $\cdot$ Interference $\cdot$ Second language $\cdot$ Long-term memory

\section{Introduction}

Studies show that resting can support memory retention (Cowan et al. 2004; Della Sala et al. 2005; Dewar et al. 2012a; for a different view see Varma et al. 2017). A brief period of rest after learning new information leads to better delayed recall performances than switching to another task, termed resting effect. Explanations for the resting effect can be found in retroactive interference and consolidation theories.

Electronic supplementary material The online version of this article (https://doi.org/10.1007/s12144-017-9760-z) contains supplementary material, which is available to authorized users.

Markus Martini

markus.martini@uibk.ac.at

Benjamin Riedlsperger

benjamin.riedlsperger@gmail.com

Thomas Maran

thomas.maran@uibk.ac.at

Pierre Sachse

pierre.sachse@uibk.ac.at

1 University of Innsbruck, Innrain 52, 6020 Innsbruck, Austria
Retroactive interference states that memory formation is disturbed by material encoded after learning of a memorandum leading to a loss of the memory content (Botvinick and Plaut 2006; Brown et al. 2007; Farrell and Lewandowsky 2002; Müller and Pilzecker 1900; Oberauer and Kliegl 2006). Accordingly, longer time lags between learning and distraction result in better recall performances than shorter time lags (e.g. Brown et al. 2007; Dewar et al. 2009; Ecker et al. 2015).

With respect to consolidation theory (Wixted 2004; Wixted and Cai 2013), new information takes time to stabilize, i.e. to be transformed into longer lasting memories, and consequently to become less vulnerable to interference (Robertson 2012). Memory consolidation describes the post-encoding stabilization process of memory representations (Dudai 2004, 2012). Studies with animals and humans show that forgetting takes place when consolidation is impaired through different interventions after learning, such as administration of neurotransmitters, hormones, electrical or magnetic stimulation, reward, and shifting cognitive resources away from the consolidation process (Dewar et al. 2012a; Fischer and Born 2009; McGaugh 2015). Neuroscientific studies revealed that consolidation processes are associated with a post-learning heightened activity of brain areas that were active during the learning process itself. The strength of the post-learning rest activity is 
thereby positively related to the delayed recall performance (Tambini et al. 2010). The hypothesis is that a heightened post-learning activity during rest represents a passive "replay" of learned memory contents (Foster and Wilson 2006; Wilson and McNaughton 1994). This passive replay stabilizes the memory content making it longer lasting and probably less prone to distraction (Wixted 2004). It is assumed that a passive replay takes place during sleep and even states of quiet wakeful rest (Deuker et al. 2013; Peigneux et al. 2006; Staresina et al. 2013). Electrophysiological studies indicate that a replay during a state of sleep and quiet rest may show similar oscillatory patterns (Brokaw et al. 2016). A phase of quiet wakeful rest supports consolidation of newly learned memory contents by low amounts of external stimulus input and learning material related interference (Dewar et al. 2007, 2009, 2012a).

The resting effect was replicated several times with different (i) populations (Alber et al. 2014; Cowan et al. 2004; Dewar et al. 2009, 2012a, b), (ii) post-learning activities (Craig et al. 2014; Mercer 2015), (iii) study materials (Craig et al. 2015, 2016; Dewar et al. 2012a), and (iv) temporal intervals between immediate and delayed recall tests (Brokaw et al. 2016; Cowan et al. 2004; Dewar et al. 2012a). However, emerging evidence suggests that resting may not always be associated with beneficial effects in terms of memory retention with relevant studies showing similar memory performances over time in rest and distractor conditions (e.g. Varma et al. 2017). In this light, identifying the limits under which resting supports memory retention is essential for its purposed application.

The present study aimed at further testing the resting effect with second language learning material in a non-native English speaking student population. Since English can be considered as a global language, it affects various aspects of life in non-English natives, e.g. when reading scientific papers, visiting lectures, attending international conferences, or perceiving daily web news, videos, and messages. However, this information is often processed and shared in the first language. Accordingly, in our study, participants were asked to memorize stories in their second language (English) and recall story details in their first language (German).

\section{Experiment 1}

In our study, each participant was involved in two learning phases (Fig. 1). In the first phase, participants learned one of two stories written in participants' second language (English), followed by an immediate recall in their first language (German) and 8 min of wakeful resting. The second phase was identical to the first, except that participants now learned the second story and performed a visual distractor task. After
7 days, participants were invited for another experimental session, where a surprise free recall took place. In line with the majority of existing findings, we hypothesized to find a higher 7 days recall performance in the rest compared to the distractor condition.

\section{Methods}

\section{Participants}

Twenty-eight students ( 19 female; mean age $=23.46$ years, age range $=18-35$ years) took part in the experiment in exchange for course credit (mean A level in English $M=2.51$, $S D=.14$; approximation to the US system: A-; all participants had at least 12 years of English lessons). Participants were tested individually in in the first session $(\sim 40 \mathrm{~min})$ and in groups of maximum four in the second session $(\sim 5 \mathrm{~min})$.

\section{Materials and Procedure}

The experiment consisted of two testing sessions (Fig. 1). Session 1 included two story learning phases. Each learning phase consisted of (i) a visual presentation of one of two stories written in English with the instructions to remember as many story details as possible for subsequent immediate recall; (ii) immediate recall of the respective story in German; (iii) an 8-min phase in which participants spent either resting or working on a spot-the-difference task (visual distractor condition); and (iv) 5-min spot-the-difference task (Dewar et al. 2012a).

Session 1 In each learning phase, participants read a story written in English. Participants' first language was German. Stories were generated based on the texts of Morris et al. (1997) and Baddeley et al. (2014). Each story was about one protagonist (a woman in one story and a man in the other) who was involved in an accident and a crime scene (see supplementary material). All paragraphs were equally structured, had identical word counts, and consisted of the same number of story details.

In a pre-study, stories were rated from seven university students (mean age $=21.27$ years). Participants were asked to read the two English stories once and recall as many details as possible in German. After each story, participants answered several questions on a Likert scale ranging from 1 to 5 ( $1=$ very low/easy/short; $3=$ optimal; $5=$ very high/difficult/long). Our results showed that both stories reached optimal ratings and did not differ significantly from each other regarding (i) amount of story details to remember (story $1: M=3.09$, $S D=.831$; story $2: M=3.09, S D=.688 ; p>.999$ ), (ii) imaginability (story $1: M=3.73, S D=.647 ; p=.414$ ), (iii) story length (story $1: M=2.00, S D=.447$, story $2: M=2.18$, 
Session 1

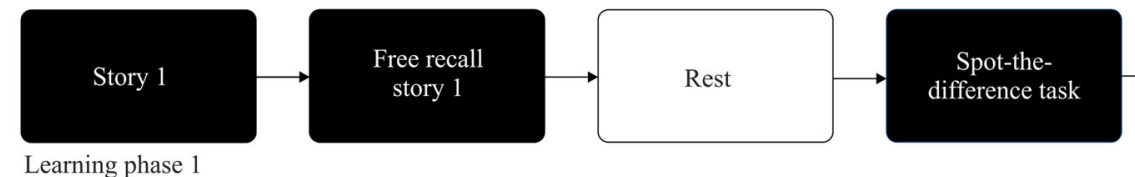

Learning phase 1

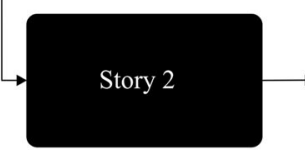

Learning phase 2

Fig. 1 Experimental procedure. In Session 1, participants learned two stories in English (second language). The critical manipulation occurred after an immediate free recall of the respective story. Participants either wakefully rested or performed a distractor task. The latter consisted of a spot-the-difference task (visual distractor condition) in Experiment 1, and

$S D=.603 ; p=.157$ ), and (iv) language complexity (story 1 : $M=2.82, S D=.982$; story $2: M=2.64, S D=.924 ; p=.577$ ).

Participants were informed that they have to read two stories in English and recall details of the respective story in German. Participants were instructed to read each story only once, in a self-paced rate. Stories were presented on a computer screen in white colour against a blue black background (font: Arial, font size: 12, line spacing: 1.0). After reading the story, participants pressed the "Next" button in the bottom middle of the screen, thereby opening a window, in which they typed all remembered story details as detailed as possible. There was no time limit for free recall. Participants pressed the "Next" button when they felt that they recalled all remembered story details. The recall phase was followed by an 8 min rest or distractor phase. In the resting condition, participants were asked to relax quietly with their eyes closed in the darkened testing room, while the experimenter left the room. In the distractor condition, participants performed a spot-thedifference task. In this task, participants were required to search for two errors in one of two pictures without time constraint, but as fast as possible until the experimenter said "stop" after 8 min. Participants marked errors with a red circle. One hundred eleven picture sets were prepared depicting landscapes, buildings, objects, and means of traffic. Each learning phase closed with a 5-min spot-the-difference task (Fig. 1; Dewar et al. 2012a).

Session 2 In Session 2, participants were required to freely recall as much details as possible from both stories of Session 1, without time constraint. Participants typed all remembered details from story 1 and 2 in German language into a text box displayed on the screen. Recall was followed by questions on expecting a recall test in Session 2 (yes/no), thinking about the stories during the rest condition 7 days ago (yes/no), and thinking about the stories during the last 7 days (yes/no). reading a New York Times (NYT) article (verbal distractor condition) in Experiment 2. The order of the stories and post-learning conditions (rest, distractor task) was fully counterbalanced across participants in Experiment 1 and 2. After 7 days, in Session 2, a surprise free recall test took place

\section{Results}

Descriptive statistics can be found in Table 1. A graphical depiction of our results can be found in Fig. 2. We conducted a repeated-measures ANOVA with the factors time of recall (immediate, 7 days) and post-learning condition (rest, visual distractor). Results revealed a significant main effect for time of recall $(\mathrm{T}), F(1,27)=106.07, p<.001$, $\eta^{2}=.797, B F 10=6.363 \mathrm{e}+16$, and a non-significant main effect for post-learning (PL) condition, $F(1,27)=2.39$, $p=.134, \eta^{2}=.081, B F 10=0.309$. The interaction $\mathrm{T} * \mathrm{PL}$ was non-significant, $F(1,27)=.23, p=.638, \eta^{2}=.008$, $B F 10$ for the $\mathrm{T}+\mathrm{PL}$ model $=3.856 \mathrm{e}+16, B F_{10}$ for the $\mathrm{T}+\mathrm{PL}+\mathrm{T} * \mathrm{PL}$ model $=1.088 \mathrm{e}-16$. These results indicate that depending on recall performance in Session 1, recall performance after 7 days dropped to a similar extent in the rest and visual distractor condition.

Post-experimental questions revealed that 9 participants expected a recall after 7 days. Five participants thought about the stories in the 7 days since Experiment 1,2 participants indicated that they repeated the words during the resting phase in Session 1, and 1 participant indicated not to remember whether he/she had repeated the story details during the resting phase. Exclusion of these participants $(n=17)$ from

Table 1 Descriptive statistics of the recalled story details $(\max =37)$ for the respective time of recall (immediate, 7 days) and post-learning condition (rest, visual distractor)

\begin{tabular}{lllll}
\hline Time of recall & Post-learning condition & Mean & SD & N \\
\hline Immediate & Rest & 22.46 & 4.73 & 28 \\
& Visual distractor & 23.25 & 5.48 & 28 \\
7 days & Rest & 14.39 & 5.95 & 28 \\
& Visual distractor & 15.64 & 5.61 & 28 \\
\hline
\end{tabular}

$S D$, standard deviation; $N$, number of participants 


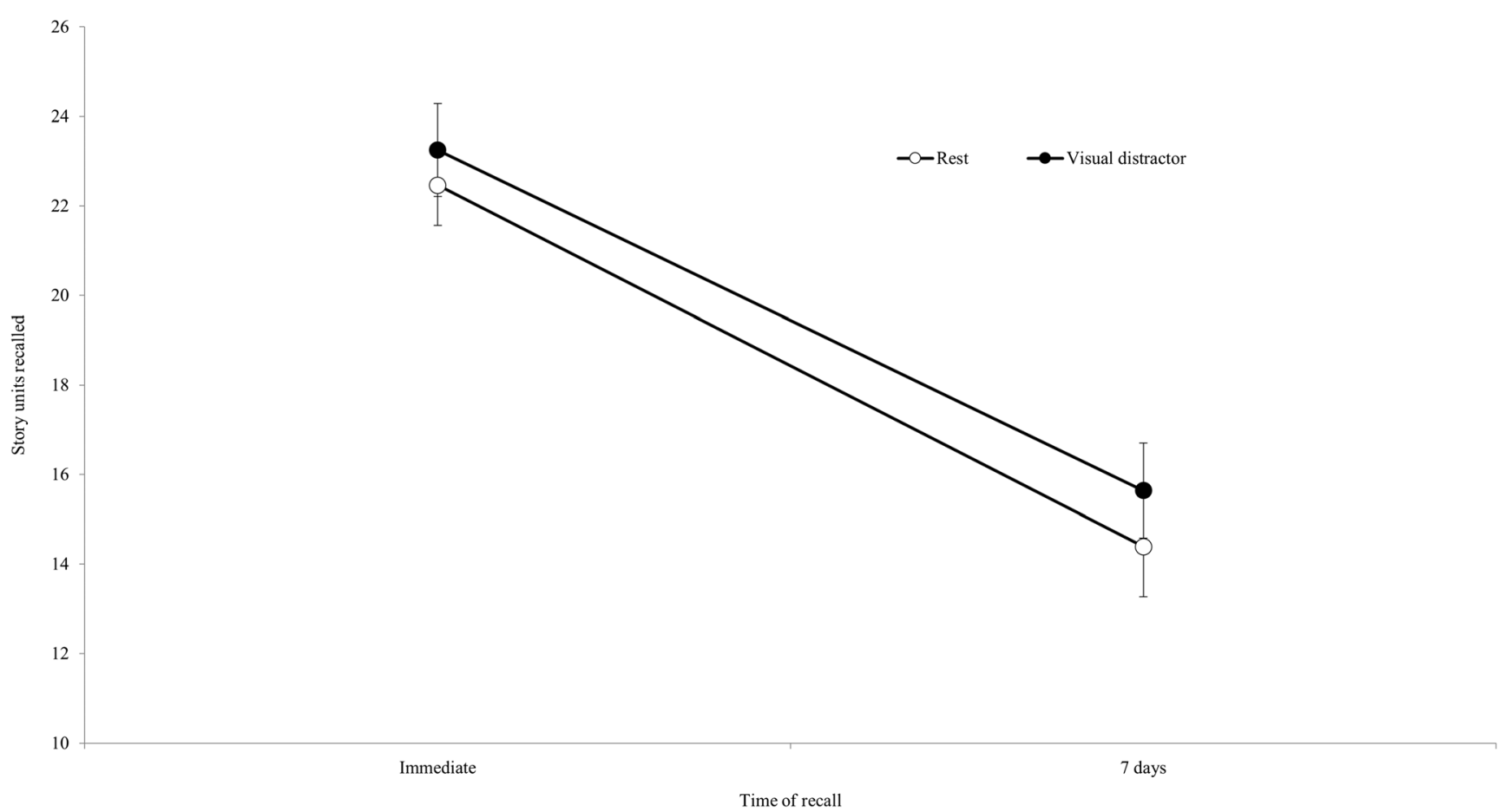

Fig. 2 Recalled story details. Depicted are the immediate and 7 days mean raw recalled story details in the rest and visual distractor condition (max $=37$ items). Error bars represent standard errors of the mean

analyses did not change results of the repeated-measures ANOVA for time of recall $(p<.001)$, post-learning condition $(p=.402)$, and time of recall*post-learning condition interaction $(p=.512)$.

Results of Experiment 1 cannot confirm that a short period of post-learning wakeful rest supports the retention of second language stories over a delay of 7 days. It is unclear, however, whether the distractor task used might have modulated this finding.

\section{Experiment 2}

Theoretical and behavioural evidence exists that any mentally effortful task after learning can disrupt memory consolidation leading to a decrease in delayed memory performance (e.g. Dewar et al. 2007, 2012a). The results of Experiment 1 did not support this view, but showed that post-learning wakeful rest was no prerequisite for successful memory consolidation (see also Varma et al. 2017). In Experiment 2, we tested whether the visual distractor task might have modulated our findings in Experiment 1. Therefore, we implemented a verbal distractor task in Experiment 2. We assumed that increasing the similarity between learning and distractor task leads to higher interference (e.g. McGeoch 1931, 1933; Müller and Pilzecker 1900) and subsequently greater differences in 7 days memory performances in the rest and distractor condition.

\section{Methods}

\section{Participants}

Thirty-six students $(24$ female; mean age $=25.67$ years, age range $=21-36$ years) took part in the experiment in exchange for course credit (mean A level in English $=2.30, S D=.27$; approximation to the US system: A-; all participants had at least 12 years of English lessons). Participants were tested individually in Session 1 ( $\sim 40 \mathrm{~min})$ and in groups of maximum four in Session 2 ( $\sim \mathrm{min})$.

\section{Materials and Procedure}

The present experimental design was identical to Experiment 1, except that participants were required to read a New York Times (NYT) article in the $8 \mathrm{~min}$ distractor condition (Fig. 1; http://www.nytimes.com/ 2013/01/22/science/earth/seeking-clues-about-sea-levelfrom-fossil-beaches.html). Content of the to-beremembered stories and the NYT article differed. The NYT article dealt with how records of past climate change can help to predict sea level rise in a world of global warming. A printed version of the article was handed out to the participants directly after the immediate recall test. The text was selected in such a way that finishing it within 8 min was not possible. Participants were asked to carefully read the article in a self-paced rate, to remember as much details as possible, and to mark their last read word when 
the experimenter said "stop". The order of the stories and post-learning conditions (rest, verbal distractor task) was fully counterbalanced across participants. For a better distinction from Experiment 1, we defined the 'NYT article phase' as verbal distractor condition, even though we are aware that both the NYT article and the spot-the-difference task are visual in nature.

\section{Results}

Descriptive statistics can be found in Table 2. A graphical depiction of our results can be found in Fig. 3. We conducted a repeated-measures ANOVA with the factors time of recall (immediate, 7 days) and post-learning condition (rest, verbal distractor). Results revealed a significant main effect for time of recall $(\mathrm{T}), F(1,35)=177.57, p<.001, \eta^{2}=.835, B F 10=$ $9.886 \mathrm{e}+19$, and a non-significant main effect for postlearning (PL) condition, $F(1,35)=.07, p=.794, \eta^{2}=.002$, $B F 10=0.203$. The interaction $\mathrm{T} * \mathrm{PL}$ was non-significant, $F(1,27)=.07, p=.791, \eta^{2}=.002, B F 10$ for the $\mathrm{T}+\mathrm{PL}$ mod$\mathrm{el}=1.834 \mathrm{e}+19, B F 10$ for the $\mathrm{T}+\mathrm{PL}+\mathrm{T} * \mathrm{PL}$ model $=$ $4.695 \mathrm{e}+18$. This result indicates that depending on recall performance in Session 1, recall performance after 7 days dropped to a similar extent in the resting and verbal distractor condition.

Post-experimental questions revealed that 14 participants expected a recall after 7 days. Four participants thought about the stories in the 7 days since Experiment 1, another 4 participants indicated that they repeated the words during the resting phase in Session 1, and 1 participant could not remember whether he/she had repeated the story details during the resting phase. Exclusion of these participants $(n=23)$ from analyses did not change results of the repeated-measures ANOVA for time of recall $(p<.001)$, post-learning condition $(p=.643)$, and time of recall*post-learning condition interaction $(p=.689)$.

Results demonstrate that an 8-min period of wakeful resting had no memory supporting effect on story retention over 7 days.

Table 2 Descriptive statistics of the recalled story details $(\max =37)$ for the respective time of recall (immediate, 7 days) and post-learning condition (rest, verbal distractor)

\begin{tabular}{lllll}
\hline Time of recall & Post-learning condition & Mean & SD & N \\
\hline Immediate & Rest & 23.19 & 5.40 & 36 \\
& Verbal distractor & 22.83 & 4.38 & 36 \\
7 days & Rest & 14.50 & 5.96 & 36 \\
& Verbal distractor & 14.42 & 6.59 & 36 \\
\hline
\end{tabular}

$S D$, standard deviation; $N$, number of participants

\section{Discussion}

The results of our study demonstrate that wakeful resting after learning had no beneficial effect on the retention of information over the long term. These findings stand in contrast to a number of previous studies investigating the resting effect under different experimental conditions (Brokaw et al. 2016; Cowan et al. 2004; Craig et al. 2014, 2015; Della Sala et al. 2005; Dewar et al. 2009, 2012a, b; Mercer 2015), but support the results of recent studies, which showed that resting is no prerequisite for successful memory consolidation (e.g. Varma et al. 2017). In the following, we address possible arguments for our findings.

One explanation is that information was processed more elaborated. 'More elaborated' is a weakly defined umbrella term subsuming various task-, situation- and personspecific conditions, which affect the way information is processed (Craik 2002; Craik and Lockhart 1972). In the present study, such factors might have been (i) no time constraint for reading and recalling the stories; (ii) unusual experimental requirements, i.e. reading a story in the second language and recalling it in the first language (Paivio 1986; Sadoski and Quast 1990); (iii) emotional arousing learning material (crime and accident scene; LaBar and Cabeza 2006); and (iv) familiar story contents that were relatable to pre-existing long-term memory knowledge (Ericsson and Kintsch 1995). Accordingly, more elaborated story processing may have led to more stable memory representations less prone to distraction (Robertson 2012), which in turn resulted in similar delayed recall scores in the rest and distractor condition.

It could be argued that wakeful resting has only a shortlived effect (minutes to hours) for second language learning material and diminishes after several days (Brown et al. 2007). In this view, 7 days recall performance should be similar in the rest and distractor condition. In our experiments, we did not include a delayed surprise free recall at the end of Session 1, as done in previous studies (e.g. Dewar et al. 2012a). The reason for this was to reduce the memory enhancing effects of an active retrieval (Roediger and Karpicke 2006), which may support rapid memory consolidation (Antony et al. 2017), and consequently would have confounded our results. According to Dewar et al. (2012a), we should have found a beneficial effect for resting, even without an intermediary recall.

The findings above show that it is an open question how a beneficial effect of resting develops over time, and to what extent it is affected by the memory content and the investigated population (e.g. younger vs. elderly). The effects of wakeful resting on verbal learning material was primarily tested over shorter retention intervals, i.e. minutes (e.g. Brokaw et al. 2016; Mercer 2015). Long-term effects (several days) of resting were found in healthy elderly adults who learned 


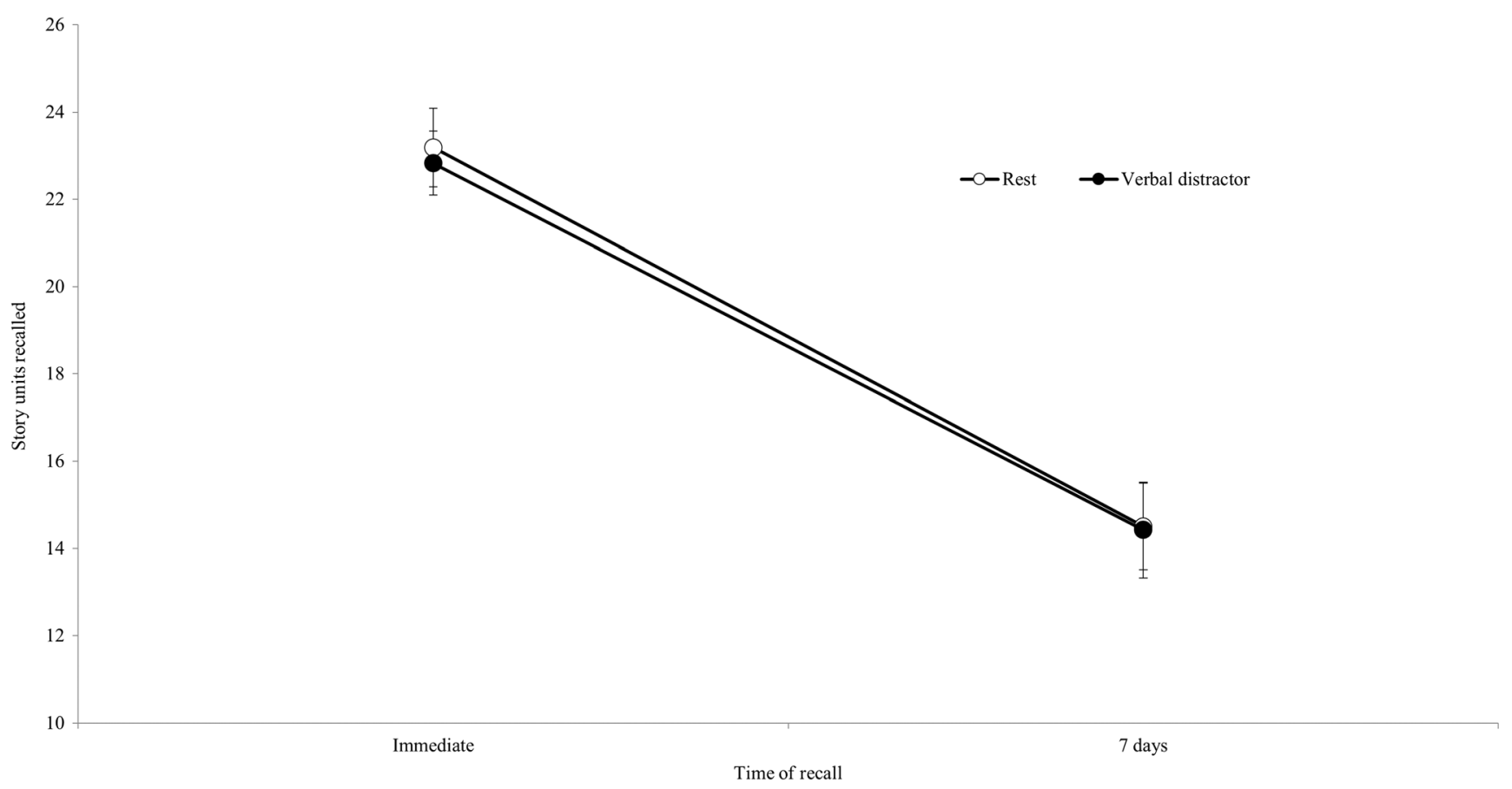

Fig. 3 Figure 2 Recalled story details. Depicted are the immediate and 7 days mean raw recalled story details in the rest and verbal distractor condition $(\max =37$ items). Error bars represent standard errors of the mean

details of stories (Dewar et al. 2012a), and in younger adults who were required to memorize spatial associative and temporal order information (Craig et al. 2015).

Furthermore, it could be argued that a resting period of $8 \mathrm{~min}$ was too short. We assume that $8 \mathrm{~min}$ are adequate to detect an existing effect, since various studies found the resting effect over shorter (minutes to hours) and longer retention intervals (days) with varying post-learning resting periods between 8 to 12 min (Brokaw et al. 2016; Craig et al. 2014; Dewar et al. 2012a, b; Mercer 2015). Furthermore, results of Dewar et al. (2009) indicate that the first minutes after learning seem to be crucial for a memory supporting effect of resting. The authors found that distraction is more effective at the beginning of a 9min post-learning phase (first $3 \mathrm{~min}$ ) than in the middle or at the end (last $3 \mathrm{~min}$ ). It seems that the longer a memory content can exist under low interference conditions, the more stable and resistant it becomes against interference (Brown et al. 2007; Wixted 2004). This assumption is supported by earlier studies of Müller and Pilzecker (1900) showing that a 6-min interval between two word lists was sufficient to protect memories of the first list from interference of the second list.

It is an open question what causes higher rates of forgetting in the post-learning interference condition. Existing studies implemented different post-learning distractors like spot-thedifference tasks (Dewar et al. 2012a), games (Brokaw et al. 2016), memorization of words and faces (Mercer 2015), all showing a resting effect. However, only recently, results of a study by Varma et al. (2017) showed no beneficial effect of resting. Over six experiments, the authors found similar delayed recall performances in the post-learning rest and distractor condition, even when varying different study parameters like the difficulty of the distractor task. Varma et al. argue that when the post-learning activity has minimal semantic and hippocampally-based episodic memory processing demands, consolidation in the distractor condition should be equally effective as in the rest condition. Similarly, Schlichting and Bäuml (2016) found an absence of forgetting with a passive wakeful rest period (hearing emotionally neutral music or perceiving neutral pictures) between learning and recall, but only when participants were required to directly forget the word list after its presentation. However, when participants were required to remember the word list, resting had no effect.

Future research has to identify relevant conditions under which resting is beneficial and interference is detrimental for memory consolidation, respectively. Moreover, not only the distractor, but also the resting state may affect the effectiveness of a brief period of rest. It is assumed that during resting processes like monitoring, mental imagination, and mind wandering take place. Accordingly, resting is not a passive cognitive state, but consumes mental resources, which may be required for memory consolidation processes. A study by Craig et al. (2014) indicates that intensive mind wandering has a negative impact on memory consolidation, in that autobiographical thinking interferes with episodic memories in the resting phase. 


\section{Conclusion}

The present study showed that a brief period of wakeful rest after learning had no beneficial effect on memory retention over the long term in non-native English speaking students. Our results provide deeper insight into specific conditions under which resting can support memory retention. Further research is needed to derive practical implications for different learning settings.

Acknowledgements Open access funding provided by University of Innsbruck and Medical University of Innsbruck.

\section{Compliance with Ethical Standards}

Conflict of Interest The authors declare no conflict of interest.

Informed Consent Informed consent was obtained from all participants. The present research was conducted with approval by the Institutional Review Board of the university.

Open Access This article is distributed under the terms of the Creative Commons Attribution 4.0 International License (http:// creativecommons.org/licenses/by/4.0/), which permits unrestricted use, distribution, and reproduction in any medium, provided you give appropriate credit to the original author(s) and the source, provide a link to the Creative Commons license, and indicate if changes were made.

\section{References}

Alber, J., Della Sala, S., \& Dewar, M. (2014). Minimizing interference with early consolidation boosts 7 -day retention in amnesics patients. Neuropsychology, 28(5), 667-675.

Antony, J. W., Ferreira, C. S., Norman, K. A., \& Wimber, M. (2017). Retrieval as a fast route to memory consolidation. Trends in Cognitive Sciences, 21, 573-576.

Baddeley, A., Rawlings, B., \& Hayes, A. (2014). Constrained prose recall and the assessment of long-term forgetting: The case of ageing and the crimes test. Memory, 22(8), 1052-1059.

Botvinick, M. M., \& Plaut, D. C. (2006). Short-term memory for serial order: A recurrent neural network model. Psychological Review, 113(2), 201-233.

Brokaw, K., Tishler, W., Manceor, S., Hamilton, K., Gaulden, A., Parr, E., \& Wamsley, E. J. (2016). Resting state EEG correlates of memory consolidation. Neurobiology of Learning and Memory, 130, 17-25.

Brown, G. D. A., Neath, I., \& Chater, N. (2007). A temporal ratio model of memory. Psychological Review, 114(3), 539-576.

Cowan, N., Beschin, N., \& Della Sala, S. (2004). Verbal recall in amnesiacs under conditions of diminished retroactive interference. Brain, 127(4), 825-834.

Craig, M., Della Sala, S., \& Dewar, M. (2014). Autobiographical thinking interferes with episodic memory consolidation. PLoS One, 9(4), e93915.

Craig, M., Dewar, M., Della Sala, S., \& Wolbers, T. (2015). Rest boosts the long-term retention of spatial associative and temporal order information. Hippocampus, 25(9), 1017-1027.

Craig, M., Dewar, M., Harris, M. A., Della Sala, S., \& Wolbers, T. (2016). Wakeful rest promotes the integration of spatial memories into accurate cognitive maps. Hippocampus, 26(2), 185-193.

Craik, F. I. M. (2002). Levels of processing: Past, present ... and future? Memory, 10(5-6), 305-318.
Craik, F. I. M., \& Lockhart, R. S. (1972). Levels of processing: A framework for memory research. Journal of Verbal Learning and Verbal Behavior, 11(6), 671-684.

Della Sala, S., Cowan, N., Beschin, N., \& Perini, M. (2005). Just lying there, remembering. Improving recall of prose in amnesic patients with mild cognitive impairment by minimising interference. Memory, 13(3-4), 435-440.

Deuker, L., Olligs, J., Fell, J., Kranz, T. A., Mormann, F., Montag, C., et al. (2013). Memory consolidation by replay of stimulus-specific neural activity. Journal of Neuroscience, 33(49), 19373-19383.

Dewar, M., Cowan, N., \& Della Sala, S. (2007). Forgetting due to retroactive interference: A fusion of Müller and Pilzecker's (1900) early insights into everyday forgetting and recent research on anterograde amnesia. Cortex, 43, 616-634.

Dewar, M., Garcia, Y. F., Cowan, N., \& Della Sala, S. (2009). Delaying interference enhances memory consolidation in amnesic patients. Neuropsychology, 23(5), 627-634.

Dewar, M., Alber, J., Butler, C., Cowan, N., \& Della Sala, S. (2012a). Brief wakeful resting boosts new memories over the long term. Psychological Science, 23(9), 955-960.

Dewar, M., Pesallaccia, M., Cowan, N., Provinciali, L., \& Della Sala, S. (2012b). Insights into spared memory capacity in amnestic MCI and Alzheimer's disease via minimal interference. Brain and Cognition, 78(3), 189-199.

Dudai, Y. (2004). The neurobiology of consolidations, or, how stable is the engram? Annual Review of Psychology, 55, 51-86.

Dudai, Y. (2012). The restless engram: Consolidations never end. Annual Review of Neuroscience, 35, 227-247.

Ecker, U. K., Tay, J. X., \& Brown, G. D. (2015). Effects of prestudy and poststudy rest on memory: Support for temporal interference accounts of forgetting. Psychonomic Bulletin and Review, 22(3), $772-778$.

Ericsson, K. A., \& Kintsch, W. (1995). Long-term working memory. Psychological Review, 102(2), 211-245.

Farrell, S., \& Lewandowsky, S. (2002). An endogenous distributed model of ordering in serial recall. Psychonomic Bulletin \& Review, 9(1), 59-79.

Fischer, S., \& Born, J. (2009). Anticipated reward enhances offline learning during sleep. Journal of Experimental Psychology: Learning, Memory, and Cognition, 35(6), 1586-1593.

Foster, D. J., \& Wilson, M. A. (2006). Reverse replay of behavioural sequences in hippocampal place cells during the awake state. Nature, 440(7084), 680-683.

LaBar, K. S., \& Cabeza, R. (2006). Cognitive neuroscience of emotional memory. Nature Reviews Neuroscience, 7(1), 54-64.

McGaugh, J. L. (2015). Consolidating memories. Annual Review of Psychology, 66, 1-24.

McGeoch, J. A. (1931). The influence of four different interpolated activities upon retention. Journal of Experimental Psychology, 14(4), 400-413.

McGeoch, J. A. (1933). Studies in retroactive inhibition: II. Relationships between temporal point of interpolation, length of interval, and amount of retroactive inhibition. Journal of General Psychology, 9(1), 44-57.

Mercer, T. (2015). Wakeful rest alleviates interference-based forgetting. Memory, 23(2), 127-137.

Morris, J., Kunka, J. M., \& Rossini, E. D. (1997). Development of alternate paragraphs for the logical memory subtest of the Wechsler memory scale-revised. The Clinical Neuropsychologist, 11(4), 370-374.

Müller, G. E., \& Pilzecker, A. (1900). Experimentelle Beiträge zur Lehre vom Gedächtnis. Zeitschrift für Psychologie, Ergänzungsband, 1, $1-300$.

Oberauer, K., \& Kliegl, R. (2006). A formal model of capacity limits in working memory. Journal of Memory and Language, 55(4), 601626. 
Paivio, A. (1986). Mental representations: A dual coding approach. New York: Oxford University Press.

Peigneux, P., Orban, P., Balteau, E., Degueldre, C., Luxen, A., Laureys, S., \& Maquet, P. (2006). Offline persistence of memory-related cerebral activity during active wakefulness. PLoS Biology, 4(4), e100.

Robertson, E. M. (2012). New insights in human memory interference and consolidation. Current Biology, 22(2), R66-R71.

Roediger, H. L., \& Karpicke, J. D. (2006). Test-enhanced learning: Taking memory tests improves long-term retention. Psychological Science, 17(3), 249-255.

Sadoski, M., \& Quast, Z. (1990). Reader response and long-term recall for journalistic texts: The roles of imagery, affect, and importance. Reading Research Quarterly, 25(4), 256-272.

Schlichting, A., \& Bäuml, K.-H. T. (2016). Brief wakeful resting can eliminate directed forgetting. Memory, 25(2), 254-260.

Staresina, B. P., Alink, A., Kriegeskorte, N., \& Henson, R. N. (2013). Awake reactivation predicts memory in human. Proceedings of the
National Academy of Sciences of the United States of America, 110(52), 21159-21164.

Tambini, A., Ketz, N., \& Davachi, L. (2010). Enhanced brain correlations during rest are related to memory for recent experiences. Neuron, 65(2), 280-290.

Varma, S., Takashima, A., Krewinkel, S., van Kooten, M., Fu, L., Medendorp, W. P., et al. (2017). Non-interfering effects of active post-encoding tasks on episodic memory consolidation in humans. Frontiers in Behavioral Neuroscience, 11, 54.

Wilson, M. A., \& McNaughton, B. L. (1994). Reactivation of hippocampal ensemble memories during sleep. Science, 265(5172), 676-679.

Wixted, J. T. (2004). The psychology and neuroscience of forgetting. Annual Review of Psychology, 55, 235-269.

Wixted, J. T., \& Cai, D. J. (2013). Memory consolidation. In K. N. Ochsner \& S. Kosslyn (Eds.), The Oxford handbook of cognitive neuroscience, volume 1: Core topics. Oxford: Oxford University Press. 